\title{
Grid Refinement in Adaptive Non-rigid Registration
}

\author{
Hyunjin Park and Charles R. Meyer
}

\begin{abstract}
Department of Radiology, University of Michigan Medical School, MI 48109, USA
\{hyunjinp, cmeyer\} aumich.edu
\end{abstract}

\begin{abstract}
Non-rigid mutual information (MI) registration algorithms with many degrees of freedom (DOF) are quite useful, but they come at high computational cost and have convergence issues. As a remedy adaptive nonrigid registration algorithms, where DOF is increased adaptively (i.e. the grid is refined adaptively), have been proposed. There are at least two ways to refine a grid adaptively: one based on changes in the global measure, the other based on a local measure. We compare these two and show that a local measure method can have better sensitivity to deformations than the global measure. The local measure employed is a novel method using local entropies and local MI.
\end{abstract}

\section{Introduction}

Recent registration literature has shifted focus from rigid/affine registration to nonrigid registration with many degrees of freedom (DOF) [1]. A non-rigid registration algorithm using Mutual Information (MI) as the similarity measure and B-spline as the geometric interpolant has become widely accepted [7]. In this case, final registration involves a dense grid of regularly spaced B-splines, whose DOF are determined by the number of B-splines used. Typical DOF is in the order of hundreds to thousands. While many algorithms have demonstrated their ability to register with large DOF quite successfully [2, 7], this added flexibility requires very high computation power, and algorithms sometimes failed to converge to an appropriate solution. To remedy high computation cost and convergence issues, new non-rigid registration algorithms have emerged [3-6]. Instead of a regularly placed grid of Bsplines (or control points), new algorithms place B-splines (or control points) only where they are needed to improve the overall registration. Thus, registration results have an irregularly spaced grid with less DOF.

While adaptive registration methods may have different geometric interpolants (i.e. B-splines or radial basis function) and similarity measures, they all share a common approach, i.e., they first detect areas where registration can be improved, and then they refine the grid in those areas. While there may be many ways to detect where to refine the grid, most current techniques observe differences in a global similarity metric; we suggest that a logical alternative is the use of a local similarity metric. Rhode et al use the gradient of global MI to refine grids [3, 4]. Others use methods based on entropies $[5,6]$. In this paper, we compare two methods to refine the grid, one global and one local, and show that for the two common methods chosen, the local measure method is better suited for improving the registration. Note that for global measures, cost functions are calculated across the whole data set, whereas for local measures, cost functions are calculated only over a finite local region. We also propose a novel local mismatch measure and compare it with the Rhode's method. 


\section{Methods}

The goal of a registration is to find the best transform $\mathrm{T}$ such that both data sets are best aligned by some similarity measure. Similarity measure used here is MI. The transform $\mathrm{T}$ can be specified many ways. B-spline representation is used here. Assuming $\mathrm{x}$ is a $3 \mathrm{D}$ coordinate, thus $\mathrm{x}=\left(\mathrm{x}_{1}, \mathrm{x}_{2}, \mathrm{x}_{3}\right)$, then formulation of B-spline is the following.

$$
T\left(x_{1}, x_{2}, x_{3}\right)=\sum_{l=0}^{3} \sum_{m=0}^{3} \sum_{n=0}^{3} B_{l}(u) B_{m}(v) B_{n}(w) \phi_{i+j} \phi_{j+m} \phi_{k+n}
$$

Where $\Phi$ is the uniformly spaced grid of B-splines and $\mathrm{B}_{0}$ to $\mathrm{B}_{3}$ are the third order $\mathrm{B}$ spline polynomials. More details on notations are available [7]. Other basis functions are used to define the transform. Notably Thin plate splines (TPS) and Wu's radial basis function are used [2-4]. For B-splines, the support of the transform is local; i.e. if a point in a given grid moves locally, then the transformation changes in that local area only. Thus for a local change of a grid, the transform needs to be updated locally.

MI is used as the similarity measure under the B-spline transform. The MI used here is the classical Shannon mutual information. Basically two co-registered data sets yield a joint probability density function (PDF) with tight clusters, whereas unregistered data sets yield a joint PDF with disperse clusters. While other papers used Parzen windowing for the estimation of PDFs [1], in this paper all PDFs (both marginal and joint) are estimated by histograms with fixed bin width. Bin width of the histogram is calculated from Freedman and Diaconis [8], where the optimal bin width is chosen to minimize integrated mean squared error over all squared-integrable PDFs.

$$
h_{n}=2(I Q R) n^{-1 / 3}
$$

$h$; optimal bin width, $I Q R$; inter quartile range, $n$; number of samples.

\subsection{General Non-rigid Registration Framework}

The goal of a non-rigid registration is to find a high DOF transform that registers two data sets best according to some similarity measure. Before attempting any high order registration, a simple, low order affine registration removes any large, linear global effects. This ensures that the subsequent high order transform deals with only relatively small local transforms. After the affine registration, a high DOF registration is performed in an iterative multi-scale fashion to save computation time. For lower resolution data, i.e., sub-sampled data, a sparse grid of B-splines is used. As resolution increases, the grid becomes denser. Also within a specific resolution, different sized grids from large to small scale are used to speed convergence of the optimization. Any time grid density is increased, the new denser grid is initialized using the previously optimized sparse grid. This multi-scale grid optimization continues for a fixed number of resolutions and a fixed number of scales within a resolution, as long as the global MI from the previous optimization has increased more than a user defined threshold. Typically, at every grid refinement in $3 \mathrm{D}$, the existing grid is halved in all dimensions resulting in an 8-fold DOF increase. 


\subsection{General Adaptive Non-rigid Registration Framework}

Reuckert's B-spline non-rigid registration works well at lower DOF, but suffers major computational complexity at high DOF [7]. For a typical CT data set of $512 \times 512 \times 60$ with voxel dimension $1 \times 1 \times 5 \mathrm{~mm}^{3}$, B-splines placed every $5 \mathrm{~mm}$ results in $1,880,000$ DOF, which leads to significant computational and convergence problems. Additionally, with B-splines placed regularly everywhere, there are many B-splines placed in background areas where there is little information to judge the registration and little interest in the local result. To remedy these computational and convergence issues, adaptive non-rigid registration algorithms have been developed [3-6]. They place or use B-splines, or control points only in areas where they are needed to improve the overall registration. Thus, the registration is computed using an irregularly spaced grid having fewer DOF. Moreover, for a given DOF adaptive algorithms can allocate a dense grid of B-splines or control points to areas of interest without wasting them in backgrounds, and thus can achieve better registration accuracies. The following is the summary of the general adaptive non-rigid registration framework.

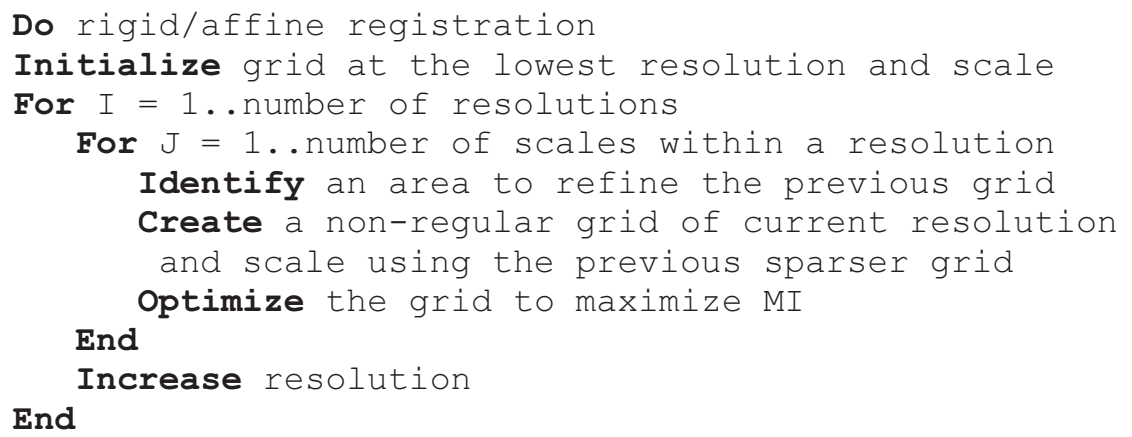

Fig. 1. General adaptive non-rigid registration algorithm

\subsection{Refinement of a Grid in Existing Adaptive Non-rigid Registration}

Rhode et al use the gradient of global MI to adaptively refine a grid [3, 4]. They use Wu's radial basis function for the geometric transform, which has a finite local support property as with B-splines, and MI as the similarity measure. The authors use the following algorithm to accomplish grid refinement. At a given scale of a grid, they put basis functions at regular intervals and then move one basis function at a time and observe the change in global MI. They argue that if the gradient of global MI is large, then the global MI is not maximum with respect to that specific location of basis function. Thus, that location is where two data sets are locally mis-registered and registration can be improved further. Once the new location of basis function is determined, 8 smaller (i.e., octree) scale basis functions occupy the area where there previously was one larger basis function. 
Rohlfing et al use B-splines and a modified similarity function where one term is the global normalized MI and the other term is the smoothness of the deformation [5]. They use local entropies to determine locally mis-registered areas and simultaneously use active B-splines and inactive (i.e., not allowed to move) B-splines to reduce DOF and effectively implement an irregular B-spline grid. Schanbel et al also use the same B-spline and two-term similarity measure, but refine their grid using local entropy, local standard deviation or gradient of the global cost function [6].

\subsection{Local Mismatch Measure M}

Local mismatches can be detected by assessing local MI. Mismatched local areas are poorly correlated by definition, and thus have low local MI. Additionally the local MI between two areas can also be low if the entropy of either of the corresponding areas is low because MI is bounded by the entropies of the individual data sets (i.e. MI(a,b) $\leq \min \{\mathrm{H}(\mathrm{a}), \mathrm{H}(\mathrm{b})\})$. Because areas of low entropy are basically featureless, they are not good candidate loci for the grid refinement. Therefore local areas of interest for the refinement of the grid are confined to those with high local entropy and low local MI. A normalized measure of mismatch $\mathrm{M}$ is introduced.

$$
M=1-\frac{M I(a, b)}{\min (H(a), H(b))}
$$

Note that all $\mathrm{MI}(\mathrm{a}, \mathrm{b}), \mathrm{H}(\mathrm{a})$ and $\mathrm{H}(\mathrm{b})$ are local measures. The mismatch measure is largest (i.e. close to 1) when MI is zero or MI is relatively smaller than $\min \{\mathrm{H}(\mathrm{a}), \mathrm{H}(\mathrm{b})\}$; also note that the mismatch measure $\mathrm{M}$ goes to zero as $\mathrm{MI}$ increases. Use of $\min \{\mathrm{H}(\mathrm{a}), \mathrm{H}(\mathrm{b})\}$ in the denominator is to provide tighter bound than $\mathrm{H}(\mathrm{a})$ or $\mathrm{H}(\mathrm{b})$ at the cost of mathematical tractability, e.g. continuity.

\section{Experiments}

Data sets used here are $\mathrm{T} 1$ and $\mathrm{T} 2$ weighted MR images from BRAINWEB [10] where the noise level is $3 \%$ in both images. The T1 slice is chosen as the reference data set and the T2 slice is the homologous data set. The homologous set is deformed locally in a known way using the following formula,

$$
d(r)=r\left(5 e^{-r / \text { rate }}+1\right)
$$

where $r$ is the radius measured from the center of subblock to be deformed, $d(r)$ is the newly assigned radius from the center of the subblock and rate is the scale at which deformation occurs. With this radial dilation deformation, the scale of deformation can be manipulated to be large or small. If the rate is large, then $d(r)$ decays slowly and thus, generates a large scale deformation. 


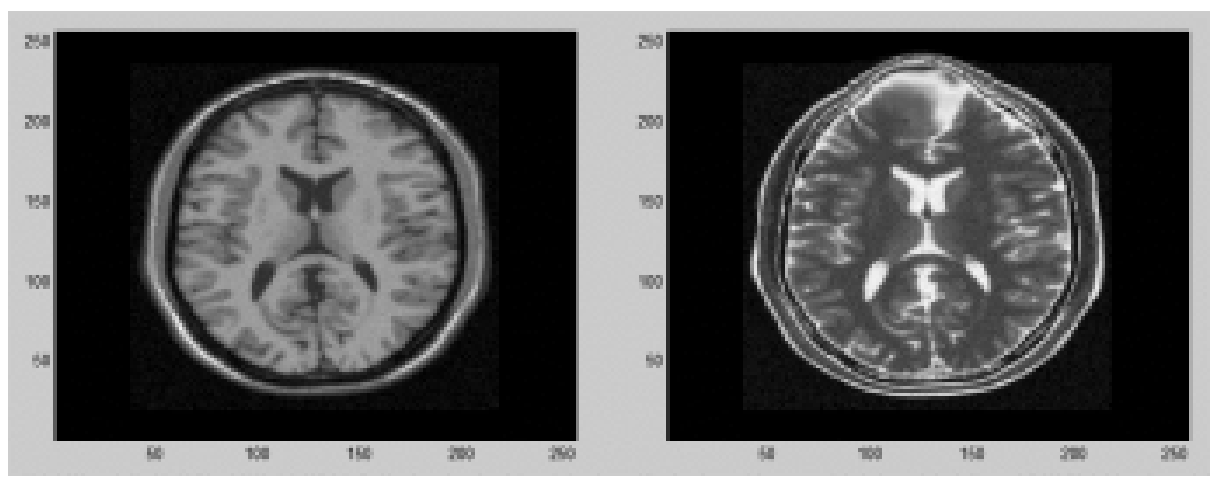

Fig. 2. Data sets used. Left: reference data set. Right: homologous data set with dilation centered at $(125,215)$.

\subsection{Sensitivity of Local vs. Global Measurements}

In this section we compare the sensitivity of two registration cost functions, local MI and global MI, with respect to various scales of deformation. Both measures can be thought of as observations of a matched filter in performing a detection task. Borrowing from standard methods to compare outputs of matched filters [6], the ratio $\mathrm{C}$ is defined as the following as a metric to compare different objective functions,

$$
\begin{aligned}
C & =\frac{\left.\frac{\partial^{2}}{\partial \theta^{2}} S(\theta)\right|_{\theta=\theta_{0}}}{\sqrt{N_{0}}}=\frac{\left.\frac{\partial^{2}}{\partial \theta^{2}} E[M I(\theta)]\right|_{\theta=\theta_{0}}}{S t d(M I(\theta))} \\
\theta_{0} & =\underset{\theta}{\arg \max } E[M I(\theta)]
\end{aligned}
$$

where $s(\theta)$ is the noiseless output, $\mathrm{N} 0$ is the noise variance, and the peak of the objective function occurs at . This ratio $\mathrm{C}$ favors objective functions with less noise and high curvature at the expected extremum point because such objective functions have a smaller error distribution of the loci of the extremum. We computed $\mathrm{C}$ by fitting the observations of global and local MI with 2nd degree polynomials and evaluating their second partials at the ideal maximum. The polynomial is assumed to be the noiseless objective function and noise is calculated from the mean of variances of MI values at different deformation scales. Here N0 includes all noise sources, and specifically the effects of local minima and maxima. The plots have been scaled and shifted to have the same noise and peak so that the signal to noise ratio differences between the two curves can be easily observed.

In this first experiment, we assume that the center of dilation in the homologous data set is known and the scale of dilation varies from small to large. Both global MI and local MI are recorded as we increase the scale of deformation. Note that local MI is confined to a subblock centered on the dilation center. Optimal bin width is applied to both measures and independent zero mean Gaussian noise is added to both reference and homologous data sets. From Fig. 3, it is evident that using local MI is 
more sensitive to local deformations than global MI. The ratio $\mathrm{C}$ for this case is 0.0020 (global MI) and 0.0038 (local MI). We observed similar trend (i.e., local MI observations have more sensitivity than global MI observations) over a range of image noise variances (e.g. 9, 25, 49 and 91). This result suggests that local measures (i.e., local MI) have better sensitivity than global measures (i.e., global MI), and are better suited for detecting local mis-registrations.

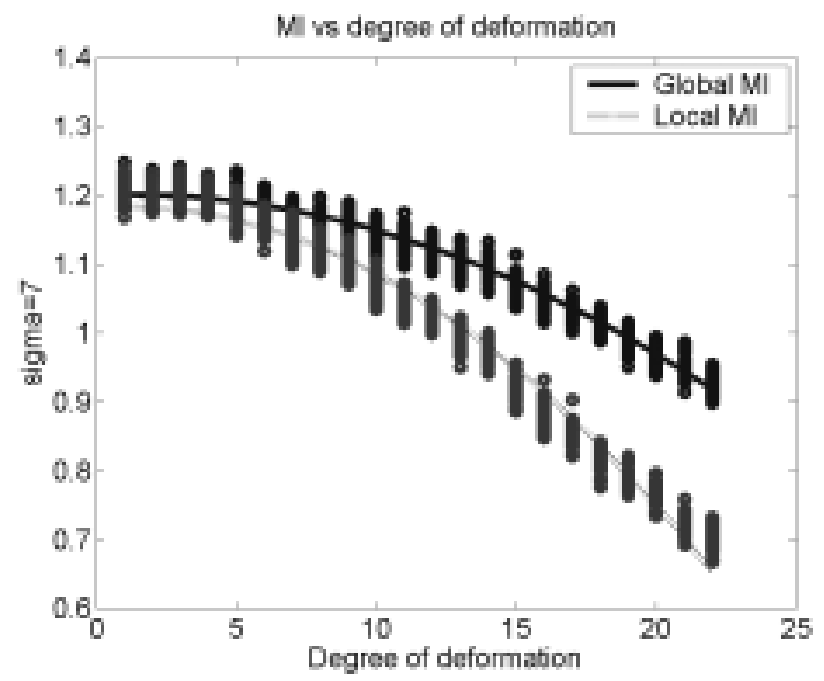

Fig. 3. Global/Local MI vs. degree of deformation. Solid line is the global MI fit and dotted line is the local MI fit. Circles are the MI values of different realization. Added noise variance of the image is 49 . Note that there are 50 realizations for each scale of deformation.

\subsection{Local Mismatch Measure vs. Gradient of Global MI}

In the second experiment, we use the more realistic scenario where the dilation center is unknown and compare the performance of our local mismatch measure and gradient of global MI. B-splines are used instead of Wu's radial basis function for evaluating the gradient of MI as described in Section 2.3. We apply a known dilation of a given scale and try to detect the center of dilation by both our local mismatch measure, M, and gradient of global MI by Rhode et al. Both mismatch measures are evaluated every 5 pixels. Subblock size and B-spline spacing are dependent on deformation scale for local mismatch measure, M, and gradient of global MI respectively. Both scale dependent parameters are chosen so that both methods have maximum ability to detect the given deformation. We have tried different scales of deformation (rate $=2,3,5$ and 8 ) have found that for large scale deformations (e.g. rate $=5,8$ ) both mismatch measures perform well, while for small scale deformations (e.g. rate $=2,3$ ) our local mismatch measure, $\mathrm{M}$, performs better. The results in Section 3.1 suggest that global measures may have less sensitivity than local measures. Although for large scale deformations the gradient of global MI has enough 
sensitivity to detect the dilation, as the deformation scale decreases, global measures may not have the sensitivity needed to detect small dilations.
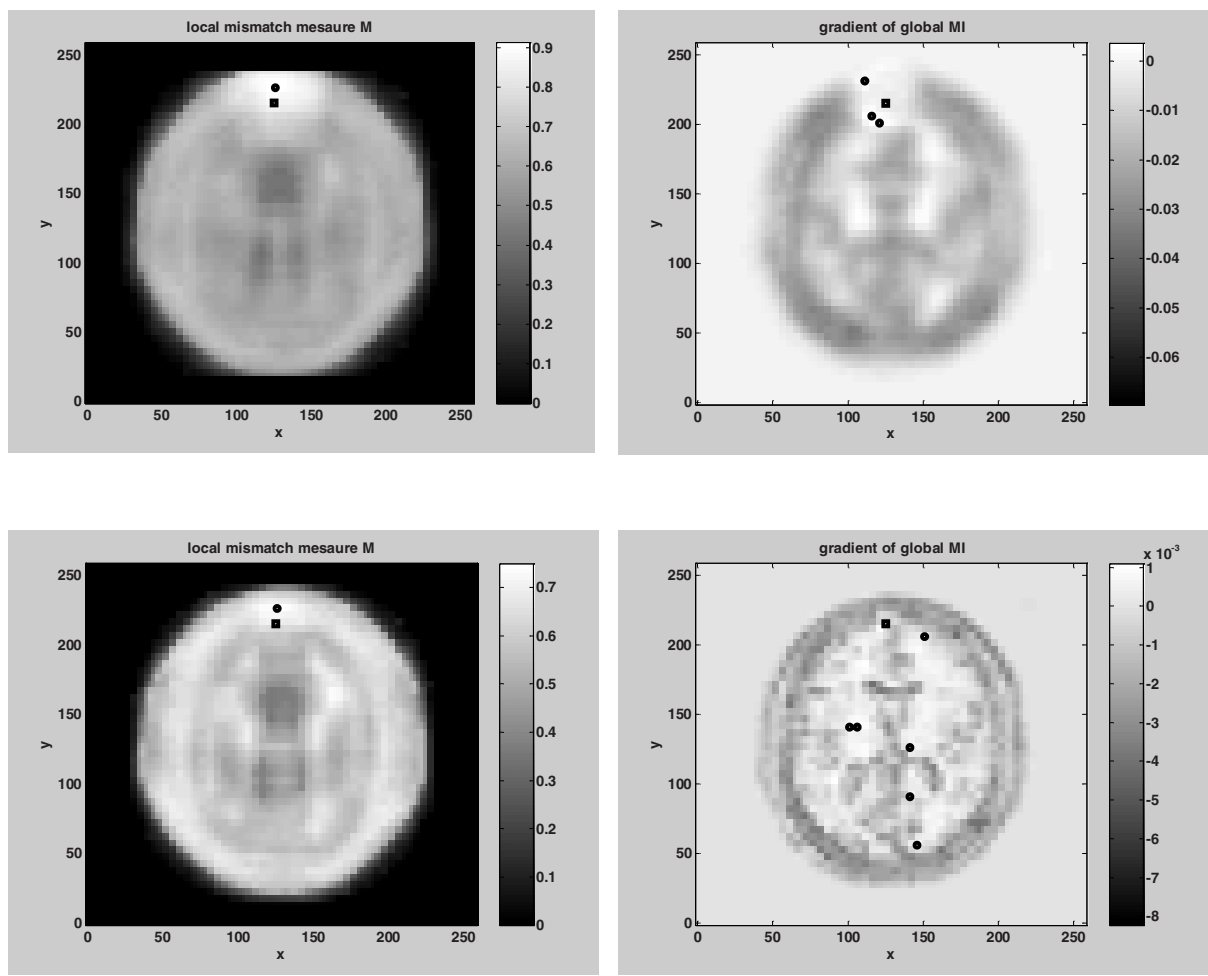

Fig. 4. Local mismatch measure $M$ vs. gradient of global MI for large and small deformations $($ rate $=5,2)$. Black box is the true dilation center and black circle is the dilation center found by mismatch measures over 5 trials. First row is the large deformation case and the second row is the small deformation case. For the large deformation, both measures perform well while for the small deformation only local mismatch measure $\mathrm{M}$ is successful.

\section{Summary}

We show a general framework in adaptive non-rigid registration algorithms and note that there are many ways to refine a grid within the adaptive non-rigid registration framework. In Section 3.1, we show that local MI is a better detector of dilation than global MI. We suggest that this is probably due to the increased sensitivity of local measurements. Though we only show this for local vs. global MI, we suggest the same trend will be observed for most pairs of local vs. global similarity functions. In Section 3.2, we show that our local mismatch measure, M, works well over a large range of deformation scale, while the gradient of global MI only works for large scale 
deformations. While the mismatch measure we present, $\mathrm{M}$, is only one of many possible mismatch measures, we suggest that grid refinement, or the detection of the locally mis-registered areas, should be based on local measures rather than global ones.

\section{References}

1. D. L. G. Hill, P. G .Batchelor, M. Holden and D. J. Hawkes, "Medical image registration," Physics in medicine and biology, vol. 46, pp. r1-r45, 2001

2. C. R. Meyer, J. L. Boes, B. Kim, P. Bland, et al., "Demonstration of accuracy and clinical versatility of mutual information for automatic multimodality image fusion using affine and thin plate spline warped geometric deformations," Medical Image Analysis, vol. 3, pp. 195-206, 1997.

3. G. K. Rhode, A. Aldroubi and B. M. Dawant, "Adaptive free form deformation for interpatient medical image registration," IPMI 2001, Proceedings of SPIE Vol. 4322, p. 15781787, 2001.

4. G. K. Rhode, A. Aldroubi and B. M. Dawant, "The adaptive bases algorithm for nonrigid image registration,” IPMI 2002, Proceeding of SPIE Vol. 4684, p. 933-944, 2002.

5. T. Rohlfing and C. R. Maurer, "Intensity based non-rigid registration using adaptive multilevel free form deformation with an incompressibility constraint," MICCAI 2001, LNCS 2208, p.111-119, 2001

6. A. Schnabel, D. Reuckert, M. Quist, et al., "A generic framework for non-rigid registration based on non-uniform multi-level free form deformations," MICCAI 2001, LNCS 2208, p.573-581, 2001.

7. D. Rueckert, L. I. Sonoda, C. Hayes, D. L. G. Hill, M. O. Leach and D. J. Hawkes, "Nonrigid registration using free-form deformations : application to breast MR images," IEEE Trans. on Medical Imaging, vol. 18, pp. 712-721, 1999.

8. A. Izenman, "Recent development in nonparametric estimation" Journal of American Statistical Association, vol. 86, Issue 413 (Mar., 1991), pp 205-224

9. M. I. Skolnik, "Introduction to radar systems," $3^{\text {rd }}$ ed., McGraw-Hill, 2000.

10. C. A. Cocosco, V. Kollokian, R. K. - S. Kwan, and A. C. Evans, "BrainWeb: Online Interface to a 3D MRI Simulated Brain Database," NeuroImage, vol.5, no.4, part 2/4, S425, 1997 